\title{
Networks of high frequency inhomogeneous transmission lines
}

\author{
F. Ossevorth ${ }^{1}$, H. G. Krauthäuser ${ }^{1}$, S. Tkachenko ${ }^{2}$, J. Nitsch ${ }^{2}$, and R. Rambousky ${ }^{3}$ \\ ${ }^{1}$ TU Dresden, Elektrotechnisches Institut, Helmholtzstraße 9, 01069 Dresden, Germany \\ ${ }^{2}$ Otto-von-Guericke-Universität Magdeburg, Lehrstuhl für Elektromagnetische Verträglichkeit, 39016 Magdeburg, Germany \\ ${ }^{3}$ Bundeswehr Research Institute for Protective Technologies and NBC Protection, Humboldtstraße 100, \\ 29633 Munster, Germany
}

Correspondence to: F. Ossevorth (fabian.ossevorth@tu-dresden.de)

Received: 13 January 2015 - Revised: 16 March 2015 - Accepted: 20 March 2015 - Published: 3 November 2015

\begin{abstract}
It is well known from classical transmission line theory, that transmission lines can be folded into impedances and thereby used in an electrical network setting. But it is also possible to create large networks of transmission lines consisting of tubes and junctions. The tubes contain the transmission lines and the junctions consider the mutual influences of the adjacent tubes or the terminals. The calculation of the currents and voltages at the junctions can be performed with the help the BLT-equation. So far this method is not applicable for nonuniform transmission lines described in a full wave method, because the lack of a distinct voltage gives no possibility for junctions. Junctions only make sense, when the considered network offers the possibility to propagate a TEM-Mode. If this requirement is fullfilled, nonuniform transmission lines could be included in an electrical network. This approach is validated in this paper in form of numerical simulations as well as measurements.
\end{abstract}

\section{Introduction}

In classical transmission line theory the input impedance of any line can be calculated at any position. Through this method the line itself can be used in an electrical network to calculate the necessary power at the load. By using the input impedance transmission lines can also be branched. Since voltage and current are distinct at every position along the line branch points can be included to combine transmission lines and to build complex networks. These branch points are called junctions and consider the mutual influences of the branched transmission lines. Such a network can be computed with the aid of the BLT-equation, as shown in Tesche et al. (1997, Sect. 6).
When the transmission line is not homogeneous the classical theory is no longer suitable. With a full wave approach all mutual effects of the line are considered, a reduction of the line through the input impedance is not accurate. For the impedance a distinct voltage is necessary, but in a full wave method only potentials exist. But under certain circumstances a nonuniform line can also be reduced, similar to a classical transmission line. It is also possible to include nonuniform lines with branches. This will be shown in the following sections.

The first section gives a brief overview of the Transmision Line Super Theory, a full wave method. In the following section parts of a nonuniform line are combined into an impedance similar to the classical theory. The results will show, that such an approach is valid. Following the collapsing of the line, a simple network with one branch will be investigated and treated numerically.

\section{Transmission Line Super Theory}

Transmission Line Super Theory (TLST) is a concise analytic method to obtain the potential and current along nonuniform wires, as described in Nitsch and Tkachenko (2010). These wires are approximated as thin wires with infinite conductivity.

Given are $n$ wires above an infinitely conducting ground plane. The scalar potential $\phi$ has the form 


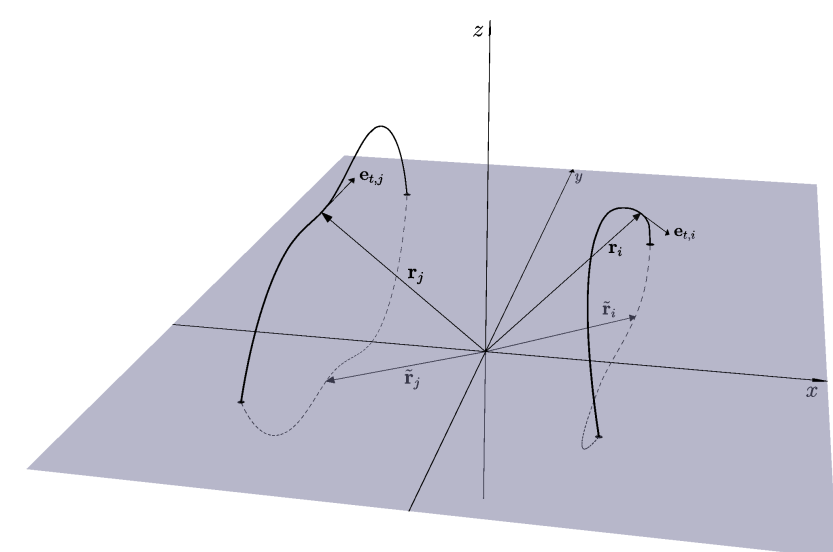

Figure 1. Arbitrary wires above groundplane.

$$
\begin{aligned}
\phi & =-\frac{1}{j \omega 4 \pi \varepsilon_{0}} \sum_{i=1}^{n} \int_{0}^{L_{i}} \frac{\partial I_{i}\left(l_{i}^{\prime}\right)}{\partial l_{i}^{\prime}}\left(G\left(l_{i}, l_{i}^{\prime}, k\right)-\widetilde{G}\left(l_{i}, l_{i}^{\prime}, k\right)\right) d l_{i}^{\prime} \\
& =-\frac{1}{j \omega 4 \pi \varepsilon_{0}} \sum_{i=1}^{n} \int_{0}^{L_{i}} \frac{\partial I_{i}\left(l_{i}^{\prime}\right)}{\partial l_{i}^{\prime}} G_{C}\left(l_{i}, l_{i}^{\prime}, k\right) d l_{i}^{\prime}
\end{aligned}
$$

where $G\left(l_{i}, l_{i}^{\prime}, k\right)$ is Green's function in free space

$G\left(l_{i}, l_{i}^{\prime}, k\right)=\frac{e^{-j k\left|\boldsymbol{r}_{i}\left(l_{i}\right)-\boldsymbol{r}_{i}^{\prime}\left(l_{i}^{\prime}\right)\right|}}{\left|\boldsymbol{r}_{i}\left(l_{i}\right)-\boldsymbol{r}_{i}^{\prime}\left(l_{i}^{\prime}\right)\right|}$

and $\widetilde{G}\left(l_{i}, l_{i}^{\prime}, k\right)$ the mirrored Green's function

$\widetilde{G}\left(l_{i}, l_{i}^{\prime}, k\right)=\frac{e^{-j k\left|\boldsymbol{r}_{i}\left(l_{i}\right)-\widetilde{\boldsymbol{r}}_{i}^{\prime}\left(l_{i}^{\prime}\right)\right|}}{\left|\boldsymbol{r}_{i}\left(l_{i}\right)-\widetilde{\boldsymbol{r}}_{i}^{\prime}\left(l_{i}^{\prime}\right)\right|}$,

Figure 1. These functions depend on the natural arc length $l_{i}$, the integration variable $l_{i}^{\prime}$ and on $k=\frac{2 \pi f}{c}$, with the speed of light $c$ and frequency $f$.

The vector potential $\boldsymbol{A}$ is also needed

$\boldsymbol{A}=\frac{\mu_{0}}{4 \pi} \sum_{i=1}^{n} \int_{0}^{L_{i}} I\left(l_{i}^{\prime}\right)\left(\boldsymbol{e}_{t, i}\left(l_{i}^{\prime}\right) G\left(l_{i}, l_{i}^{\prime}, k\right)-\widetilde{\boldsymbol{e}}_{t, i}\left(l_{i}^{\prime}\right) \widetilde{G}\left(l_{i}, l_{i}^{\prime}, k\right)\right) d l_{i}^{\prime}$,

with $\boldsymbol{e}_{t, i}$ the tangential vector of wire $i$

$\boldsymbol{e}_{t, i}=\frac{\partial \boldsymbol{r}_{i}\left(l_{i}\right)}{\partial l_{i}}$

evaluated on the surface of the wire. Inserting the equation for $\boldsymbol{A}$ (Eq. 4) into the equation for the electrical field

$\boldsymbol{E}=\boldsymbol{E}^{\mathrm{sc}}+\boldsymbol{E}^{\mathrm{exc}}=-\nabla \phi-j \omega \boldsymbol{A}+\boldsymbol{E}^{\mathrm{exc}}$

gives a general equation for the E-field. On the surface of the wire, the boundary condition is

$\boldsymbol{n}_{i} \times \boldsymbol{E}_{i}\left(\boldsymbol{r}_{\boldsymbol{i}}\left(l_{i}\right)+\boldsymbol{e}_{\perp, i}\left(l_{i}\right) a\right)=\mathbf{0}$

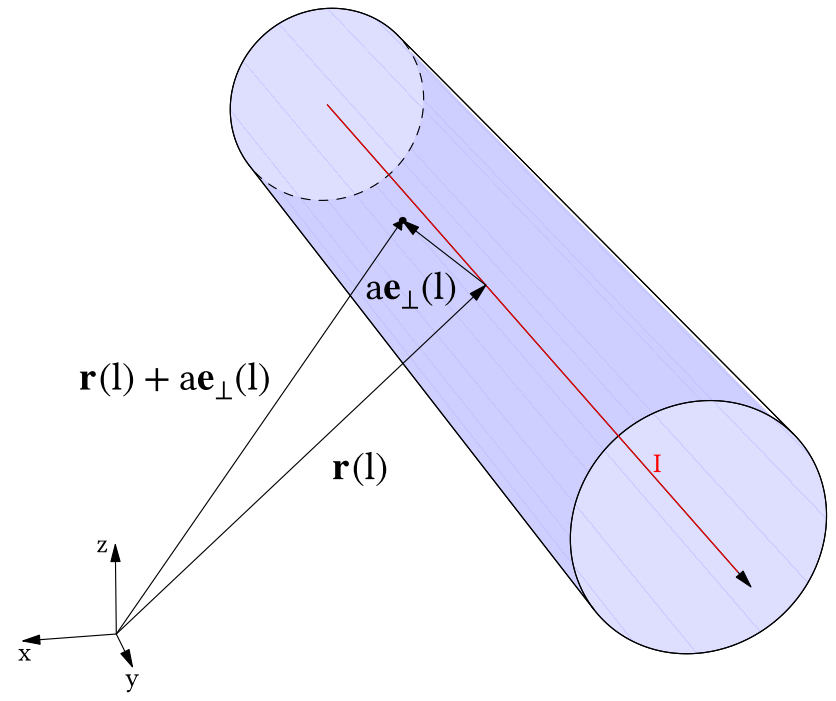

Figure 2. Point of evaluation.

with radius $a$ of the wire, Fig. 2.

If the radius $a$ is very small, then the tangential vector on the surface of the wire is very close to the tangential vector of the center of the wire. Thus, the boundary condition can be simplified to

$\boldsymbol{e}_{t, i}\left(l_{i}\right) \cdot \boldsymbol{E}_{i}\left(\boldsymbol{r}_{i}\left(l_{i}\right)\right)=0$

and it follows an integral equation for $\phi$ and $I$

$$
\begin{aligned}
& \frac{d \phi_{i}\left(l_{i}\right)}{d l_{i}}+j \omega \frac{\mu_{0}}{4 \pi} \sum_{j=1}^{n} \int_{0}^{L_{j}} I_{j}\left(l_{j}^{\prime}\right)\left(\boldsymbol{e}_{t, i}\left(l_{i}\right) \cdot \boldsymbol{e}_{t, j}\left(l_{j}^{\prime}\right) G_{i j}\left(l_{i}, l_{j}^{\prime}, k\right)\right. \\
& \left.-\boldsymbol{e}_{t, i}\left(l_{i}\right) \cdot \widetilde{\boldsymbol{e}}_{t, j}\left(l_{j}^{\prime}\right) \widetilde{G}_{i j}\left(l_{i}, l_{j}^{\prime}, k\right)\right) d l_{j}^{\prime}=\boldsymbol{E}^{\mathrm{exc}} \cdot \boldsymbol{e}_{t, i}\left(l_{i}\right) \\
& \frac{d \phi_{i}\left(l_{i}\right)}{d l_{i}}+j \omega \frac{\mu_{0}}{4 \pi} \sum_{j=1}^{n} \int_{0}^{L_{j}} I_{j}\left(l_{j}^{\prime}\right) \boldsymbol{G}_{L}\left(l_{i}, l_{i}^{\prime}, k\right) d l_{j}^{\prime} \\
& =\boldsymbol{E}^{\mathrm{exc}} \cdot \boldsymbol{e}_{t, i}\left(l_{i}\right)
\end{aligned}
$$

With the help of this equation and Eq. (1) one obtains a coupled differential equation with varying coefficients $\boldsymbol{P}$ similar to the classical transmission line equations

$$
\frac{d}{d l}\left(\begin{array}{c}
\boldsymbol{\phi}(l) \\
\boldsymbol{I}(l)
\end{array}\right)+j \omega\left(\begin{array}{ll}
\boldsymbol{P}_{11} & \boldsymbol{P}_{12} \\
\boldsymbol{P}_{21} & \boldsymbol{P}_{22}
\end{array}\right)\left(\begin{array}{c}
\boldsymbol{\phi}(l) \\
\boldsymbol{I}(l)
\end{array}\right)=\left(\begin{array}{c}
\boldsymbol{v}^{\mathrm{exc}}(l) \\
\mathbf{0}
\end{array}\right),
$$

as shown in Nitsch and Tkachenko (2010). Solving this equation gives the potential and current for every conductor with respect to the matricant, Gantmacher (1960), which depends on the common parameter $l$

$l_{i}=\frac{L_{i}}{L} l \quad 0 \leq l \leq L$, 


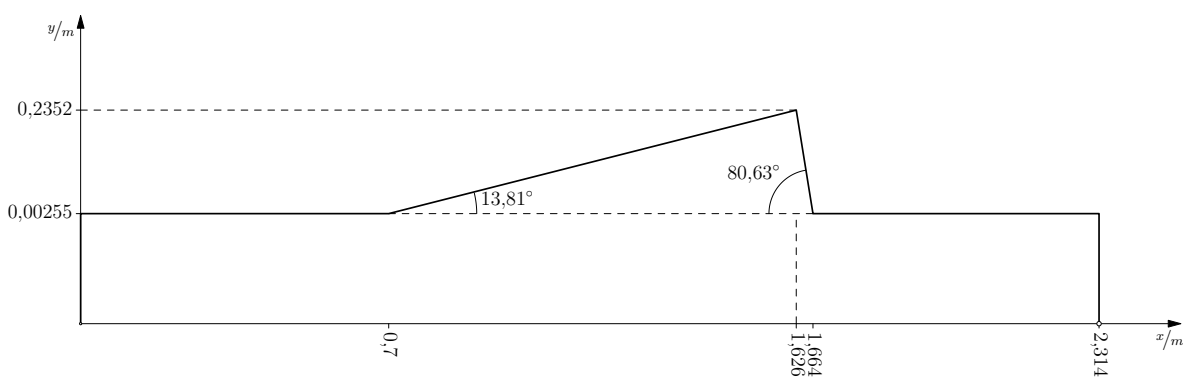

Figure 3. Geometry of a nonuniform transmission line.

giving

$$
\left(\begin{array}{c}
\boldsymbol{\phi}(l) \\
\boldsymbol{I}(l)
\end{array}\right)=\boldsymbol{M}_{l_{0}}^{l}\left(\begin{array}{c}
\boldsymbol{\phi}\left(l_{0}\right) \\
\boldsymbol{I}\left(l_{0}\right)
\end{array}\right)+\int_{l_{0}}^{l} \boldsymbol{M}_{l_{0}}^{\boldsymbol{l}^{\prime}}\left(\begin{array}{c}
\boldsymbol{v}^{\mathrm{exc}}\left(l^{\prime}\right) \\
\mathbf{0}
\end{array}\right) d l^{\prime} .
$$

The matricant is a square matrix of dimension $2 n \times 2 n$

$\boldsymbol{M}_{l_{0}}^{l}=\left(\begin{array}{ll}\boldsymbol{\phi}_{1}(l) & \boldsymbol{\phi}_{2}(l) \\ \boldsymbol{I}_{1}(l) & \boldsymbol{I}_{2}(l)\end{array}\right)$

containing two linear independent solution vectors for $\phi$ and I.

So far, the parameters are unknown. They can be determined through a perturbation approach, also shown in Nitsch and Tkachenko (2010), based on an already known solution for the matricant from classcial transmission line theory

$\boldsymbol{P}=-\frac{1}{j \omega} \frac{d}{d l} \boldsymbol{M}_{l_{0}}^{l}\left(\boldsymbol{M}_{l_{0}}^{l}\right)^{-1}$

giving an analytic solution for the parameters

$$
\begin{aligned}
& \boldsymbol{P}(l)=\frac{\mu_{0}}{4 \pi}\left(\begin{array}{cc}
\int_{l_{0}}^{L} \boldsymbol{G}_{L}\left(l, l^{\prime}, k\right) \cdot \operatorname{diag}\left(e^{-j k l_{i}\left(l^{\prime}\right)}\right) d l^{\prime} & \int_{l_{0}}^{L} \boldsymbol{G}_{L}\left(l, l^{\prime}, k\right) \cdot \operatorname{diag}\left(e^{j k l_{i}\left(l^{\prime}\right)}\right) d l^{\prime} \\
\frac{4 \pi k}{\omega \mu_{0}} \operatorname{diag}\left(e^{-j k l_{i}\left(l^{\prime}\right)}\right) & -\frac{4 \pi k}{\omega \mu_{0}} \operatorname{diag}\left(e^{j k k_{i}\left(l^{\prime}\right)}\right)
\end{array}\right)
\end{aligned}
$$

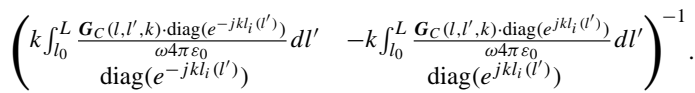

After evaluating the parameters the differential Eq. (10) can be solved with the help of the product integral, Gantmacher (1960)

$\boldsymbol{M}_{l_{0}}^{l}=\lim _{\Delta \mathfrak{t}_{k} \rightarrow 0} \prod_{k} e^{\boldsymbol{P} \Delta l_{k}}$.

Various numerical methods for the solution of this integral have been investigated in Steinmetz (2006), following these investigations a Runge-Kutta method of 4th order gives very accurate results both in computation time and accuracy of the solution.

\section{Network description of a nonuniform transmission line}

One essential part of a network description of transmission lines is the concept of the input impedance. When it is possible to collapse parts of a wire into an input impedance and use this impedance as termination for the rest of the wire, then the prerequisites for more complex networks are met.

An experiment was conducted in order to proof simulation data with measurements. A thin wire with radius $0.35 \mathrm{~mm}$ was fixed as in Fig. 3 above a conducting ground plane.

The nonuniform part in the center of the wire is flanked by uniform parts to ensure the propagation of a TEM-mode along these parts of the line. The actual structure of the wire is shown in Fig. 4.

For the measurements a network analyzer was used, connected to the line from below the ground plane. As can be seen from Fig. 5 the results of the measurements for the input impedance and the calculation match up to $200 \mathrm{MHz}$. The following deviation in the measurements is due to sideeffects of the experiment, e.g. the coupling to surrounding apertures, which is not considered in the calculations. But especially the dielectric material seems to have a considerable effect on the final results. Nevertheless, the results of a method of moments simulation and a TLST method show very good agreement. With these correct results for the complete line, new calculations for the input impedance can be compared to now proofed results.

At first the line was cut into two parts in the last half of the line and thus partitioned into a front and back part, which were calculated separately, Fig. 6 . This means, that the mutual influences between the two parts were neglected, which is an essential property of a network characteristic.

Thereafter the input impedance is calculated from Eq. (17), taking into account the terminal impedance $Z_{L}$ and the matricant of the last part of the line $\boldsymbol{M}_{3, L_{2}}^{L}$

$i(L)\left(\begin{array}{c}Z_{L} \\ 1\end{array}\right)=\boldsymbol{M}_{3, L_{2}}^{L}\left(\begin{array}{c}\phi\left(L_{2}\right) \\ i\left(L_{2}\right)\end{array}\right)$.

The separation of the line took place in the uniform part of the line, where a TEM-mode propagates, giving an explicit 


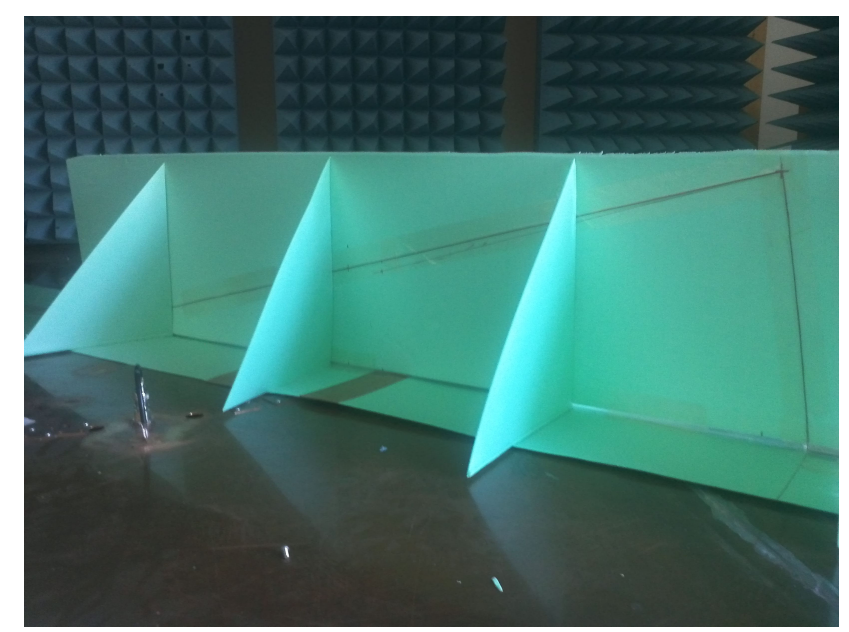

(a) Structure

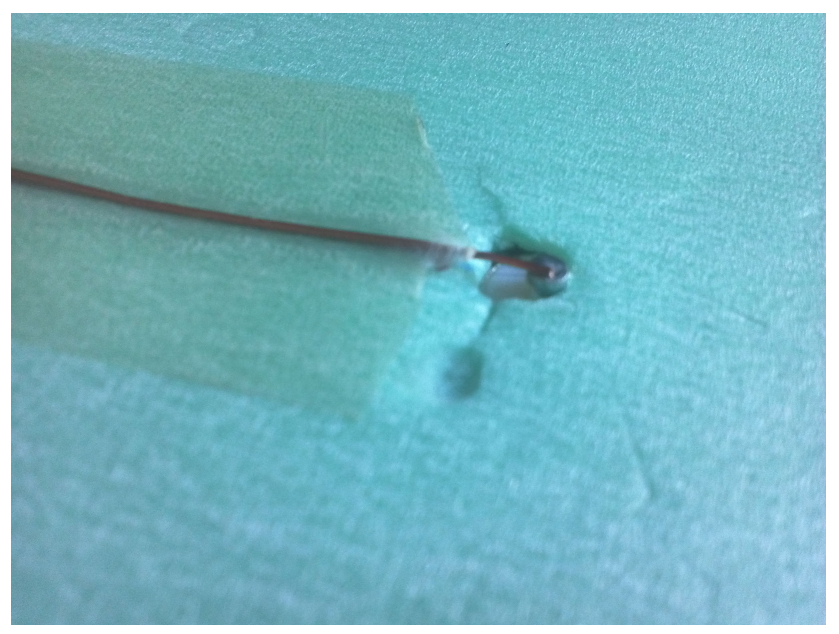

(b) Connection

Figure 4. Photographs of the real wire.

voltage and therefore the ratio between $\frac{\phi\left(L_{2}\right)}{i\left(L_{2}\right)}$ is valid giving

$Z_{i, 2}=\frac{\phi\left(L_{2}\right)}{i\left(L_{2}\right)}=\frac{M_{3,12}-Z_{L} M_{3,22}}{Z_{L} M_{3,21}-M_{3,11}}$

When cutting the line into two parts and calculating both lines separately one makes an error, when using a full wave method to compute potential and current. Since a TEM-mode is dominant on these parts of the line the parameters of the separated lines should reflect this effect. But when using the full wave method the lines end at the cutted parts and therefore the parameters also change at the end of the line. This can be avoided in the calculation of Eq. (15) by taking the lines longer than they actually are. Now, the lines seem to overlap the junction and the parameters become constant at the position where the real line ends. In this way the parameters are matched to the TEM-mode. The results show an excellent agreement which can be seen from Fig. 7.
Table 1. Layout of the network structure.

\begin{tabular}{|c|c|c|}
\hline Part & Description & \\
\hline 1 & front part & $0-0.318 \mathrm{~m}$ \\
\hline 2 & junction & $\begin{array}{l}\text { classical transmission lines } \\
\text { of length } 0.1 \mathrm{~m}\end{array}$ \\
\hline 3 & nonuniform part & $0.518-2.314 \mathrm{~m}$ \\
\hline 4 & branch & $0.324 \mathrm{~m}$ \\
\hline
\end{tabular}

\section{Branched network}

In the previous section it was shown that a nonuniform line can be calculated piecewise, when the separation of the wire took place in the uniform parts. Through this method it is shown that a nonuniform line with uniform parts can be treated as a simple network. A more complex network would include junctions too. In extending the line from the previous section about an additional wire rectangular to the line, Fig. 8, a branch point is inserted and a more complex network created.

The whole structure is now divided into four parts, Table 1. At first, each structure except the junction itself is calculated with the TLST. After that the resulting matricants are prepared for a classical electrical network solver. For that the matricants are transformed into admittance form. It is necessary to pay attention to the specific definition for the admittance transformation for each network solver. In the present case the following definition is used, Fig. 9,

$i(L)=-i_{2}, i(0)=i_{1}$ und $v(L)=v_{2}, v(0)=v_{1}$,

and the matricants are transformed with

$\overline{\boldsymbol{Y}}=\left(\begin{array}{cc}-\boldsymbol{M}_{12}^{-1} \boldsymbol{M}_{11} & \boldsymbol{M}_{12}^{-1} \\ \boldsymbol{M}_{22} \boldsymbol{M}_{12}^{-1} \boldsymbol{M}_{11}-\boldsymbol{M}_{21} & -\boldsymbol{M}_{22} \boldsymbol{M}_{12}^{-1}\end{array}\right)$.

Now each part, except for the junction, is in admittance form, which gives a reduced scheme as shown in Fig. 10. This scheme is then used for the network solver in form of Listing 1 . A voltage source of $1 \mathrm{~V}$ with a resistance of $50 \Omega$ is connected through the knots 0,1 and 2 . The current in this branch is measured with a voltage source of $0 \mathrm{~V}$. The junction is modeled with the nodes $4,5,6,8$ using three classical transmission lines with the network solver's own functions. Every line is terminated with $50 \Omega$ which reflects the connection of the networkanalyzer used for measurements. The calculated, simulated and measured results are shown in Fig. 11. The calculation and the simulation with a method of moments show good agreement. Again the measurements agree up to $400 \mathrm{MHz}$ and diverge for higher frequencies. The effects of the insulation are not considered in both the calculation and the simulation. Nonetheless, the plots show, that networks with branches can be calculated with the TLST. It is required that the branches occur in the uniform parts of a line, where a TEM-Mode can dominate and classical transmission line theory is applicable. 

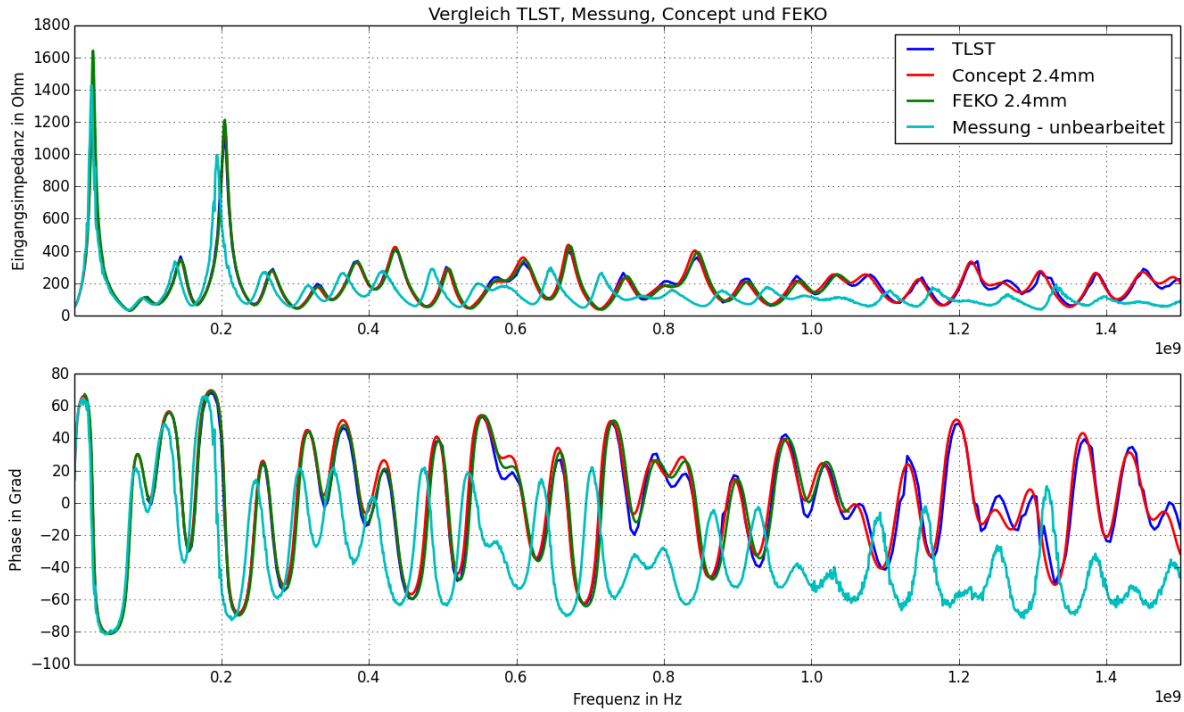

Figure 5. Magnitude and phase of the input impedance of the whole line.

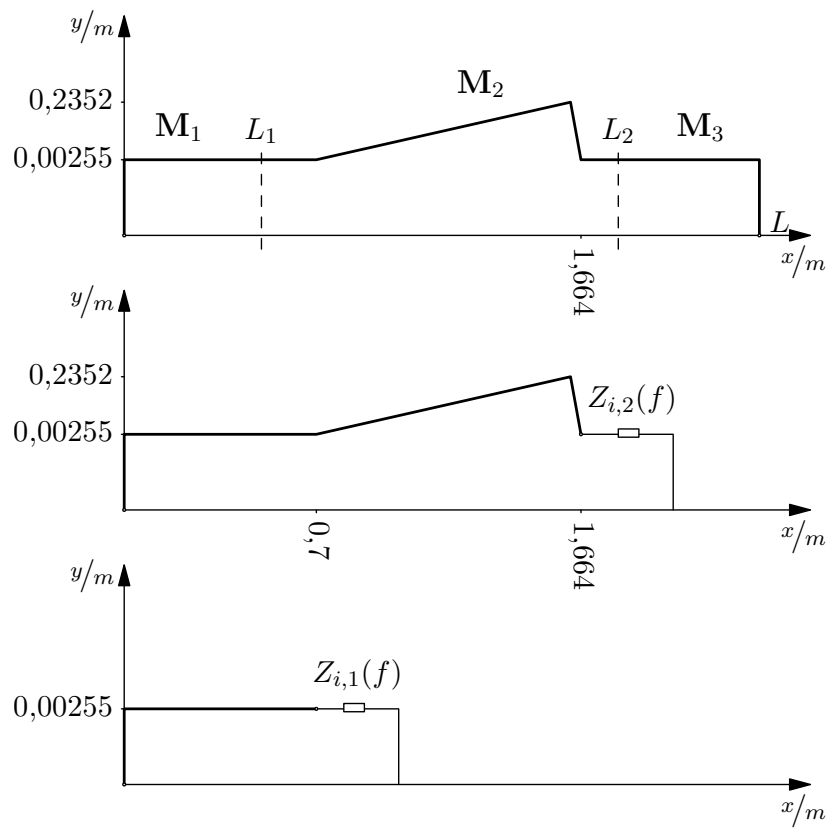

Figure 6. Calculation of input impedances

\section{Conclusions}

It could be shown in this article that nonuniform transmission lines can be divided into separate parts and calculated independently from each other. This gives the possibility for a network abstraction of nonuniform lines which can be extended to complex networks. The requirement for this approach of separating the transmission line is, that the line has uniform parts on which a TEM-mode can propagate. It is along these parts of the line that the wire can be separated

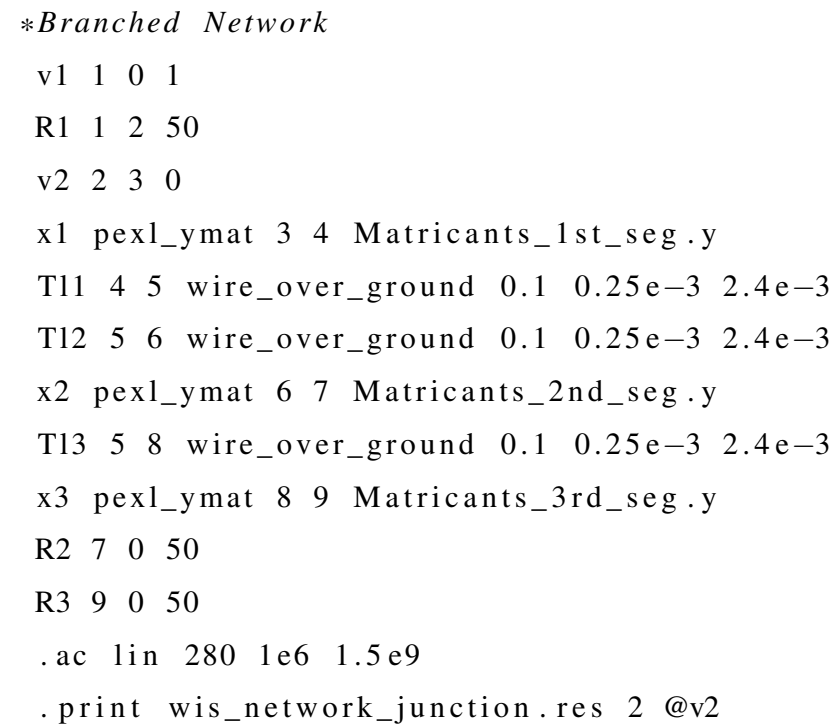

Listing 1. Script for network solver.

only. Because of the TEM-mode distinct voltages exist which allow the application of classical network theory on these parts. Apart from separating one transmission line only, there was also a branched transmission line investigated. The computed results for the single parts have been used in a network solver as admittance matrices. The overall result of the input impedance has been compared to results of a simulation with a method of moments as well as with measurements and has shown a good agreement. These results confirm the usability of the Transmission Line Super Theory for networks. Further studies are necessary to apply the theory to more complex networks with multiple conductors and to reduce the computational effort. 

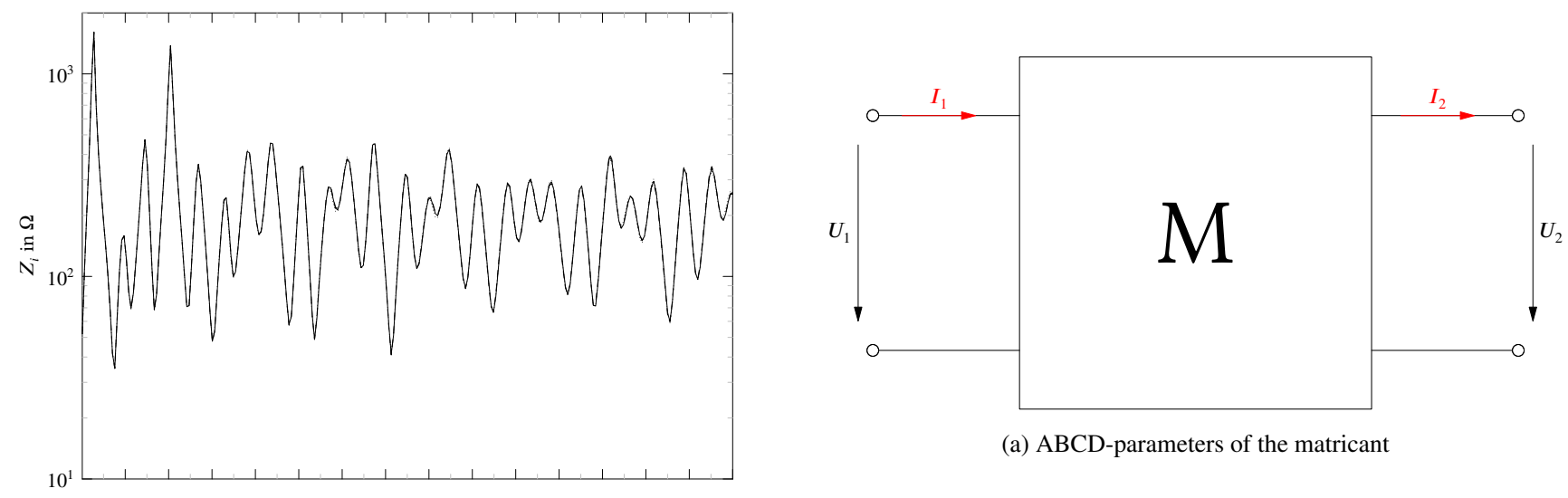

(a) ABCD-parameters of the matricant
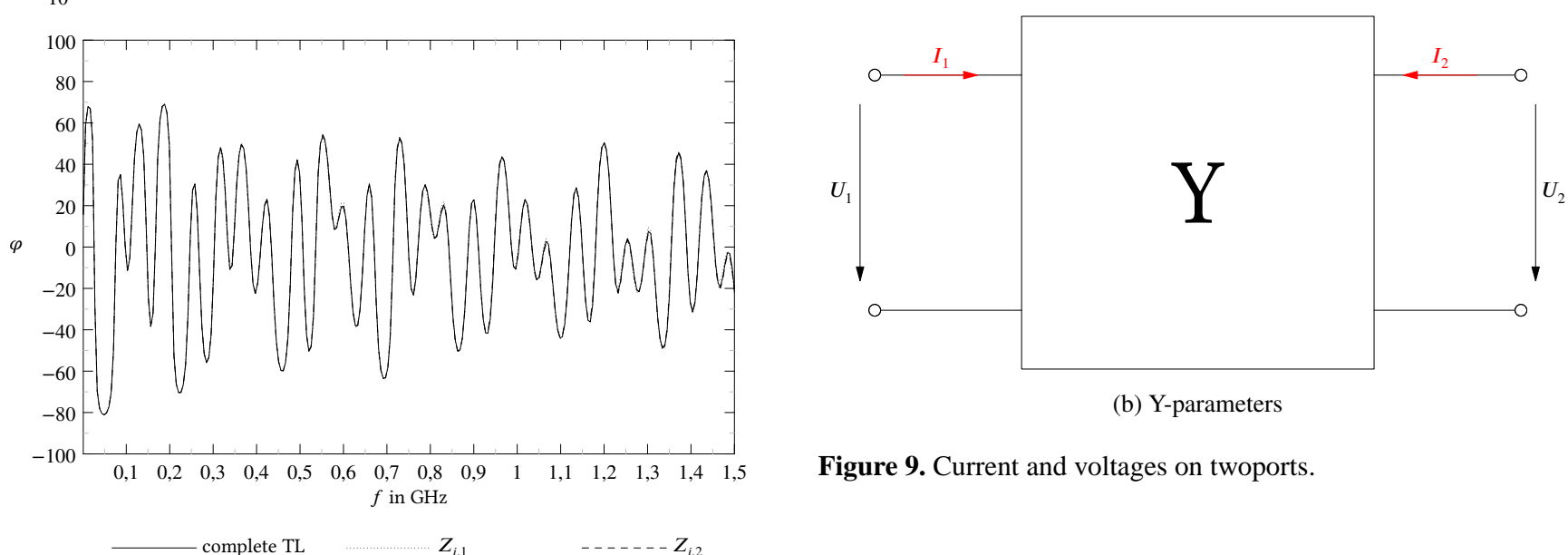

(b) Y-parameters

Figure 9. Current and voltages on twoports.

Figure 7. Results of the input impedance for the separated line with an asymptotic approach at the cutted ends.
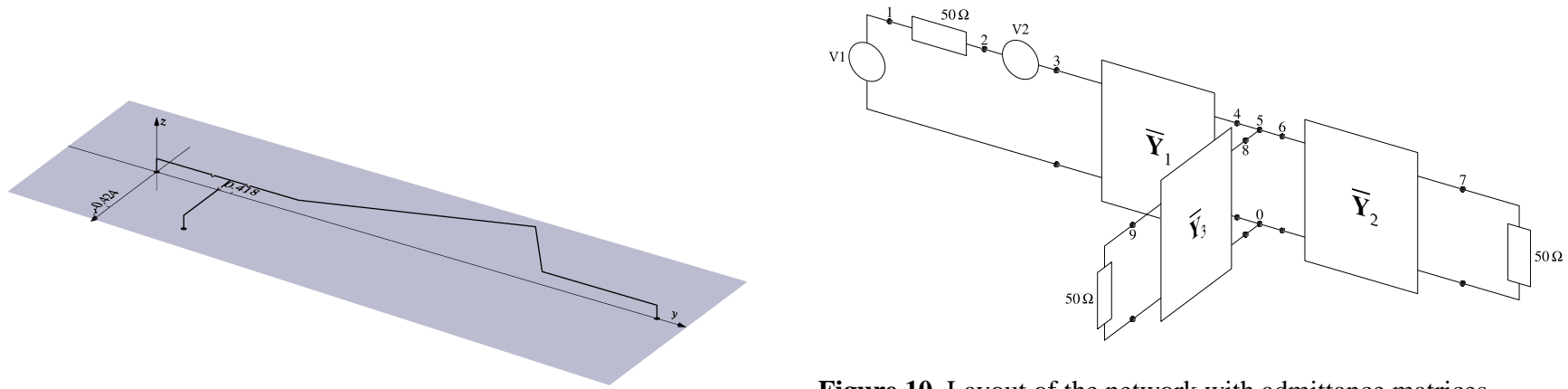

Figure 8. Network with junction

Figure 10. Layout of the network with admittance matrices. 

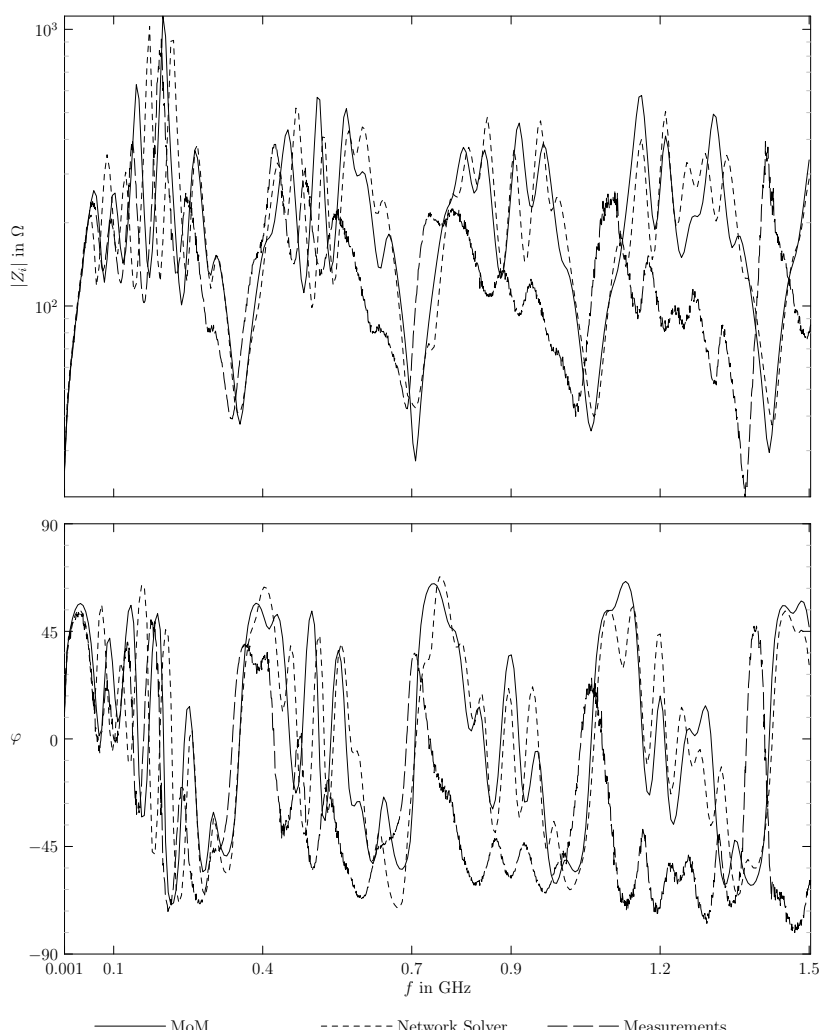

Figure 11. Input impedance on knot 3.

Acknowledgements. The research was funded by the Wehrwissenschaftliches Institut für Schutztechnologien, Munster, Germany, under the contract number E/E590/DZ005/AF119.

Edited by: F. Gronwald

Reviewed by: M. Koch and one anonymous referee

\section{References}

Gantmacher, F.: The Theory of Matrices, no. Bd. 2 in Chelsea Publishing Series, American Mathematical Society, 1960.

Nitsch, J. and Tkachenko, S.: High-Frequency Multiconductor Transmission-Line Theory, Found. Phys., 40, 1231-1252, doi:10.1007/s10701-010-9443-1, 2010.

Steinmetz, T.: Ungleichförmige und zufällig geführte Mehrfachleitungen in komplexen technischen Systemen, $\mathrm{PhD}$ thesis, Ottovon-Guericke-Universität Magdeburg, 2006.

Tesche, F. M., Ianoz, M. V., and Karlsson, T.: EMC Analysis Methods and Computational Models, John Wiley \& Sons, 1997. 